\section{Niveles de autoestima y uso de estrategias de afrontamiento en un grupo de personas privadas de la libertad en un centro de reclusión de la ciudad de Bucaramanga / Colombia ${ }^{3}$}

\author{
Richard Larrota \\ Mg. Doctorando en Psicología \\ Universidad Pontificia Bolivariana \\ Bucaramanga, Colombia \\ Correo: richardcastt@gmail.com \\ Leidy Sánchez Rojas \\ Psicóloga \\ Universidad Pontificia Bolivariana \\ Bucaramanga, Colombia \\ Correo: leidyjadirarojasquintero@gmail.com \\ Jahaira Sánchez \\ Especialista en Psicología Clínica \\ Universidad Pontificia Bolivariana \\ Bucaramanga, Colombia \\ Correo: jahairasapra@gmail.com
}

Recibido: 11/04/2015 Evaluado: 19/02/2016 Aceptado: 19/02/2016

\title{
Resumen
}

Objetivo: identificar aquellas estrategias utilizadas por los sujetos ante situaciones percibidas como estresantes, además de correlacionar la autoestima con las estrategias de afrontamiento. Método: estudio de tipo descriptivo-correlacional que evaluó la autoestima a través del Inventario de Autoestima de Coopersmith, el cual sostiene coeficientes de confiabilidad y validez entre .81 y .93. Además, se examinó el uso de estrategias de afrontamiento, mediante la Escala de Estrategias de Afrontamiento Modificada, EEC-M, la cual maneja un alfa de Cronbach de .847. Estas pruebas fueron administradas a un grupo de 41 personas detenidas en el Establecimiento Penitenciario de Mediana Seguridad y Carcelario de Bucaramanga por delitos diversos. Resultados: se evidencia que el nivel total de autoestima se sitúa cualitativamente como normal según punto de corte de 15,78 en la escala general; sin embargo, en las subescalas general, social y familia-hogar de la prueba, se evidenciaron resultados con tendencia a la baja. Por otra parte, se observó el uso frecuente y alta puntuación en las estrategias de afrontamiento que refieren a Solución de Problemas, Religión y Reevaluación Positiva. Conclusión: existen correlaciones significativas $(p<.05)$ de tipo negativo entre la autoestima y las estrategias de afrontamiento de Espera, Evitación Emocional, Reacción Agresiva y Negación. 


\section{Levels of self-esteem and use of coping strategies in a group of persons deprived of liberty in a detention center in the city of Bucaramanga / Colombia \\ Abstract}

Objective: To identify those strategies used by subjects in situations perceived as stressful, and to correlate selfesteem with coping strategies. Method: Study of the descriptive correlational kind that assessed self-esteem through the Coopersmith Self-Esteem Inventory, which supports reliability and validity coefficients between .81 and .93. Furthermore, the use of coping strategies was examined by the Coping Strategies Scale Modified, EEC-M, which manages a Cronbach's alpha of .847. These tests were administered to a group of 41 people arrested in the Medium Security Penitentiary and Prison of Bucaramanga for various offenses .. Results: it is evident that the total level of self-esteem is qualitatively placed as normal according to a cutoff of 15.78 in the general scale. However, in general, social and family-home test results subscales downward trend was evident. Furthermore, frequent use and high score on the coping strategies that refer to Troubleshooting, Religion and positive reappraisal, was observed Conclusion: there are significant correlations $(p<.05)$ of negative type between self-esteem and strategies of Waiting coping, Emotional Avoidance, Aggressive Reaction and Denial.

\section{Níveis de autoestima e uso de estratégias de afrontamento num grupo de pessoas privadas da liberdade num centro de reclusão da cidade de Bucaramanga / Colômbia}

Resumo

Objetivo: Identificar aquelas estratégias utilizadas pelos sujeitos ante situações percebidas como estressantes, ademais se correlacionar a autoestima com as estratégias de afrontamento. Método: estudo de tipo descritivo-correlacional avaliou a autoestima através do Inventário de Autoestima de Coopersmith, o qual sustem coeficientes de confiabilidade e validez entre .81 e .93. Ademais, se examinou o uso de estratégias de afrontamento, mediante a Escala de Estratégias de Afrontamento Modificada, EEC-M, a qual maneja um alfa de Cronbach de .847. Estas provas foram administradas a um grupo de 41 pessoas detidas no Estabelecimento Penitenciário de Mediana Seguridade e Prisional de Bucaramanga por delitos diversos. Resultados: se evidencia que o nível total de autoestima se situa qualitativamente como normal segundo ponto de corte de 15,78 na escala geral, porém, nas sub-escalas geral, social e família-lar da prova se evidenciaram resultados com tendência à baixa. Por outra parte, se observou 0 uso frequente e alta pontuação nas estratégias de afrontamento que referem a Solução de Problemas, Religião e Re-avaliação Positiva, Conclusão: existem correlaciones significativas $(p<.05)$ de tipo negativo entre a autoestima e as estratégias de afrontamento de Espera, Prevenção Emocional, Reação Agressiva e Negação. 


\section{ntroducción}

"Para cualquier especie animal, particularmente cuanto más desarrollada tenga la parte del cerebro referida al mundo emocional y al cognitivo, un cambio forzado de ecosistema supone un trauma importante de gravísimas consecuencias" (Segovia, 2005 p. 1). De esta manera, el Homo Sapiens Sapiens, por ser la especie animal más desarrollada en la escala evolutiva, experimentará con más rigor los cambios de hábitat a los que fuere expuesto. Por ende, la prisión como hábitat antinatural, que confina al encarcelamiento "reviste carácter de un evento traumático para la vida de una persona, especialmente si es el primer contacto con la prisión" (Ruiz, 1999 p. 1). Lo anterior, debido a factores como el dejar la convivencia familiar, la pérdida del estilo de vida habitual, las rutinas sociolaborales y de ocio, la inserción en un medio restrictivo y de privaciones y el aislamiento social (Ruiz, 2007). Sumado, a la estigmatización y a la censura social que ocasiona la reclusión en un centro penitenciario o ser ex-convicto (FernándezBessa 2006).

Así, el contexto carcelario es un medio que genera tensión, entre otros efectos psicológicos y físicos negativos nombrados ampliamente en la literatura (Herrera \& Expósito, 2010; Montenegro \& Ortega, 2008; Monteverde, 1982; Pérez \& Redondo, 1991; Ruiz, 2007; Wheeler, 2007).

A partir de estas razones, se genera el presente estudio acerca las estrategias de afrontamiento y los niveles de autoestima en personas que experimentan la situación de privación de la libertad, pues solo desde los conocimientos emitidos por la academia será factible plantear acciones en pro de la promoción de la calidad de vida enfocadas a impactar positivamente el proceso personal de resocialización de cada sujeto. Este primer acercamiento a la realidad psicológica de los sujetos en cuanto a la autoestima y a las estrategias de afrontamiento, podría contribuir a posteriores procesos de intervención penitenciaria.

Consecuente con el tema planteado, Musitu y Vera (2000), definen la autoestima como la valoración y consideración que el individuo realiza y mantiene de sí mismo como persona; de manera complementaria, Santrock (2002) manifiesta que la autoestima puede ser evidenciada, en la confianza, la autoimagen y la satisfacción que el individuo exprese de sí. Al respecto, Maslow (1982) expuso la autoestima como una necesidad para llegar a la autorrealización del ser, así, los sujetos necesitan del amor hacia sí mismos y de aquel procedente de otras personas, para autorrealizarse. Por tanto, aquellas personas quienes por diferentes motivos no han construido un nivel adecuado de estima, estando por ende, insatisfecha esta necesidad, se les dificultará el camino hacia la autorrealización y la autorrealización misma. En palabras de Branden (1995) una autoestima sólida permitirá alcanzar a la persona más rápidamente su autorrealización.

Al respecto, siendo la autoestima según Coopersmith (1981, citado por Villasmil, 2010) una disposición de 
aceptación o rechazo de un individuo hacia su propia persona, está directamente relacionada e influye en procesos psicológicos superiores tales como la planeación del futuro, las estrategias para enfrentar situaciones estresantes, la toma de decisiones, la solución de problemas, las relaciones interpersonales, la autoevaluación de sus capacidades y habilidades (Eisenberg \& Patterson, 1981 citado por Naranjo, 2007). Por lo anterior Coopersmith (1981) hizo referencia a que la autoestima permea el comportamiento de las personas, e relación con su desarrollo (clasificada por este autor como alta, media y baja). En ese sentido, observó que las personas con alta autoestima son expresivos, así como creativos y propensos a logros en sus diversas esferas de actuación, no ceden ante las críticas y perciben sus aproximaciones a los demás como positivas; por su parte, sujetos con autoestima media, si bien guardan características similares a los de alta autoestima, experimentan autoafirmaciones con moderación. Finalmente, este mismo autor, afirma que aquellos con autoestima baja se ven expuestos a problemas del estado del ánimo; con dificultades para reconocer sus cualidades. Con una marcada tendencia a percibir rechazo por parte de los otros, lo que dificulta sus relaciones.

En evidencia de lo anterior, se hace comprensible la importancia y función transcendental de la autovalía en variadas áreas de la vida de las personas, pues, como lo mencionan Cava, Musitu y Vera (2000) la autoestima sostiene relación significativa con indicadores de adaptación, bienestar psicológico y social.

En esa línea de ideas, con respecto a la autoestima en relación con la prisión, en 1973, Smith y Hogan plantearon que la prisión afecta significativamente la autovalía de la persona, generándose evaluaciones negativas de la autoimagen forjándose niveles bajos de autoestima y autopercepción; este planteamiento coincide, con los hallazgos de Marcuello y García (2011) quienes encontraron que los usos y costumbres dentro de la cárcel son interiorizados progresivamente formando niveles de autoestima bajos. En consecuencia, frente al fenómeno de prisionalización, los sujetos deberán implementar procesos adaptativos que le permitan, mantener el statu-quo, la adecuada convivencia dentro de la comunidad y la gestión adecuada de las dificultades que se presenten durante su permanencia en el establecimiento de reclusión. Por ello, las estrategias personales de afrontamiento ante situaciones estresantes, intimidantes, difíciles o adversas cobrarán importancia, pues el individuo deberá entonces movilizar sus recursos comportamentales y cognitivos para manejarlas, resolverlas y salir bien librado de ellas (Lázarus \& Folkman, 1986). Estas estrategias son llamadas de coping o afrontamiento, entendidas aquí, como toda acción realizada por cada individuo frente a situaciones de tensión, es decir, la forma o las respuestas que la persona asume para enfrentarse a las diversas situaciones tensionantes (Martín, Jiménez \& Fernández-Abascal, 1997). En palabras de Lázarus y Folkman (1986) y Fernández-Abascal y Palmero (1999) citado por Londoño, Henao, Puerta, Posada, Arango, Aguirre (2006) son todos aquellos esfuerzos cognoscitivos y conductuales realizados por los sujetos tendientes a dar solución, minimizar y/o extinguir el problema o neutralizar y/o controlar la respuesta emocional o 
reevaluar el evento. Así, en estudios realizados sobre este tema Kirchner y Mohino (2003) observaron que "los presos no siempre cuentan con unas estrategias de afrontamiento suficientemente efectivas para afrontar esta situación [de encarcelamiento] que exige una fuerte activación de recursos" (p. 2). También, Zamble y Porporino (1990) encontraron que los internos hacen un mayor uso de estrategias de afrontamiento centradas en la emoción y uno menor de aquellas centradas en el problema; lo que coincide con el hallazgo de Ruiz (2007) quien encontró que "una mayor sintomatología emocional se asocia con un mayor afrontamiento evitativo, una evaluación más negativa del clima emocional [...]" (p. 12).

Por otra parte, Greg, Justine y Roger (2002), citados por Quintanilla, Valadez, Valencia y González (2005) en coincidencia con Kirchner y Mohino (2003) hallaron que las personas privadas de la libertad que utilizan la autolesión como estrategia para afrontar y / o reducir el estrés, usan estrategias de coping menos efectivas en comparación con la población normal. En ese sentido, es importante inquirir sobre las mismas para observar aquellas estrategias de afrontamiento y niveles de autoestima que están presentes en el grupo de personas privadas de la libertad que hicieron parte del presente estudio. También, reviste relevancia indagar sobre la relación entre la variable autoestima y el uso de estrategias de afrontamiento, con el propósito de generar resultados de utilidad para el abordaje terapéutico, por parte de instituciones interesadas, de manera que se pueda favorecer el proceso de resocialización.

\section{Método}

\section{Participantes}

La muestra estuvo compuesta por 41 hombres privados de la libertad, recluidos en el Establecimiento Penitenciario de Mediana Seguridad y Carcelario de Bucaramanga (EPMSC), con edades comprendidas entre los 20 y 60 años, con una media de 30.86; quienes fueron escogidos por conveniencia.

\section{Instrumentos}

Para llevar a cabo el presente estudio, se aplicó el Inventario de Autoestima de Coopersmith, (1981) instrumento que ha sido utilizado ampliamente en el contexto colombiano, sosteniendo coeficientes de confiabilidad y validez entre 0,81 y 0,93 . Y se encuentra constituido por 58 afirmaciones con las que las personas se identifican o no, teniendo como opciones de respuesta "igual que yo" o "distinto a mí". Evalúa cuatro dimensiones de autoestima en la vida del sujeto:

1. Autoestima General: autovalía integral que el sujeto tiene de sí mismo.

2. Autoestima Social: explora el nivel en que la persona percibe ser aceptada por los pares e integrada como parte de un grupo, y su capacidad para enfrentar con éxito diferentes situaciones sociales.

3. Autoestima Familia-Hogar, representa el autovalor y autopercepción 
que se da a sí mismo el sujeto teniendo como referente la relación con su microsistema. Incluye todos aquellos sentimientos de aceptación que percibe por parte de su familia.

4. Autoestima Escolar/Laboral: autopercepción sobre capacidad para enfrentar con éxito situaciones de la vida académica-laboral específicamente rendir bien y ajustarse a las exigencias del medio. El Inventario de Autoestima de Coopersmith, permite emitir una calificación total general de autoestima del sujeto.

Además, se aplicó la Escala de Estrategias de Coping Modificada (EECM), la cual es resultado de la modificación a la escala EEC-R de Chorot y Sandín, (1993) y se encuentra adaptada y validada en Colombia (Londoño, Henao, Puerta, Posada, Arango, \& Aguirre, 2006). La escala consiste en un inventario de 69 ítems que evalúan 12 Estrategias de Afrontamiento ante las diferentes situaciones estresantes: Solución de Problemas(SP); Búsqueda de Apoyo social(BAS); Espera(ESP); Religión (REL); Evitación Emocionalᄀ (EE); Búsqueda de Apoyo Profesional (BAP); Reacción Agresiva (RA); Evitación Cognitiva (EC); Reevaluación Positiva (RP); Expresión de la Dificultad de Afrontamiento (EDA); Negación (NEG) y finalmente Autonomía (AUT). Los evaluados respondieron cada ítem de acuerdo a una escala tipo likert que tiene opciones entre "1" (nunca) a "6" (siempre). Así, las estrategias de coping son medidas a partir de las puntuaciones obtenidas. "El alfa de Cronbach de la prueba definitiva fue de 0,847" (Londoño, Henao, Puerta, Posada, Arango, \& Aguirre, 2006 p.328).

\section{Procedimiento}

En primer momento se presentó la propuesta de investigación ante la Dirección Regional Oriente del Instituto Nacional Penitenciario y Carcelario y esta a su vez remitió oficio de presentación al Establecimiento Penitenciario de Mediana Seguridad y Carcelario de Bucaramanga. Una vez aceptada la iniciativa, se tomó una muestra a conveniencia de la población y asimismo se explicó e informó a los posibles sujetos participantes sobre la investigación y su proceso. Se dió inicio a la aplicación de los instrumentos: Inventario de Autoestima de Coopersmith y la Escala de Estrategias de Coping Modificada, posterior a la firma del consentimiento informado, el cual enfatizó en la voluntad que le asistía a cada uno de los convocados para elegir si participaban o no de la investigación. En esta misma línea el proyecto contempló que los sujetos estarían expuestos a riesgos mínimos, de acuerdo a las condiciones éticas para la investigación con humanos. Finalmente, tras obtenerse los datos se realizó la sistematización de los resultados en el programa SPSS.

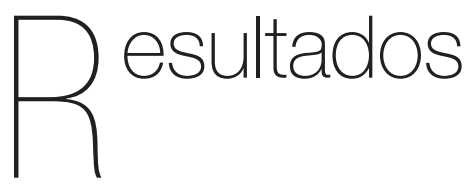

En la Tabla 1 se presentan las características sociodemográficas de la muestra, El 100\% de los participantes son hombres. En relación al estrato socioeconómico, la mayor parte de los sujetos (68.3\%) se agrupa en el nivel 1 y 2 (bajo). En cuanto al nivel educativo, el porcentaje mayor (36.6\%) tiene bachillerato 
completo, seguido de estudios básicos en secundaria incompletos $(28,8 \%)$ y primaria completa e incompleta (29.3\%). A nivel de la variable estado civil, se observó que la mayoría de sujetos viven en unión libre $(53,7 \%)$ seguido de un porcentaje significativo de sujetos solteros (36,6\%). Respecto a la religión, la mayoría de sujetos dijeron ser católicos (68,3\%). Realizan actividades escolares-laborales en el área de escuelas de formación empresarial entre estas, marroquinería, maderas, tejidos y bisuterías

Tabla 1.

Características Sociodemográficas de la muestra

\begin{tabular}{|c|c|c|}
\hline \multicolumn{3}{|c|}{ Fi (\%) } \\
\hline \multicolumn{2}{|c|}{ Género Hombre } & \multirow{2}{*}{$\begin{array}{c}41(100 \%) \\
15(36.6 \%)\end{array}$} \\
\hline Estrato & 1 & \\
\hline \multirow[t]{4}{*}{ Socioeconómico } & 2 & $13(31,7 \%)$ \\
\hline & 3 & $07(17,1 \%)$ \\
\hline & 4 & $05(12,2 \%)$ \\
\hline & 6 & $01(2,4 \%)$ \\
\hline \multirow[t]{5}{*}{ Estado Civil } & Soltero & $15(36,6 \%)$ \\
\hline & Casado & $02(4,9 \%)$ \\
\hline & Unión Libre & $22(53,7 \%)$ \\
\hline & Separado & $01(2,4 \%)$ \\
\hline & Viudo & $01(2,4 \%)$ \\
\hline \multirow[t]{6}{*}{ Escolaridad } & Primaria Incompleta & $05(12,2 \%)$ \\
\hline & Primaria Completa & $07(17,1 \%)$ \\
\hline & Bachillerato Incompleto & $11(26,8 \%)$ \\
\hline & Bachillerato Completo & $15(36,6 \%)$ \\
\hline & Técnico & $02(4,9 \%)$ \\
\hline & Magíster & $01(2,4 \%)$ \\
\hline \multirow[t]{5}{*}{ Religión } & Católico & $28(68,3 \%)$ \\
\hline & Evangélico & $06(14,6 \%)$ \\
\hline & Protestante & $01(2,4 \%)$ \\
\hline & Otras & $02(4,9 \%)$ \\
\hline & Ninguna & $04(9,8 \%)$ \\
\hline
\end{tabular}

Nota. Fuente: Análisis de datos en Statistical Package for the Social Sciences

En la Tabla 2 se evidencia, el porcentaje medio, los puntajes mínimos y máximos, el error típico de la media y la desviación típica de cada una de las 4 dimensiones de autoestima que mide la prueba, de la escala de mentira, y la puntuación general total de autoestima. En cuanto a los puntajes de cada dimensión de la prueba se obtuvo que la Autoestima General se encuentra en una media de 15,78 lo cual indica que los sujetos de la muestra manifiestan autoestima hacia sí mismos en un nivel normal o medio. Sin embargo, téngase en cuenta que esta puntuación se encuentra sobre su límite, es decir, se observa tendencia al nivel bajo. Así, este resultado a la luz de su interpretación, pone de manifiesto que aunque los sujetos presentan autovaloración y confianza en sí mismos aunado a la seguridad personal y respeto de si, dentro de parámetros estándar, son propensos a experimentar desánimo, tristeza profunda, preocupación, sensibilidad a las críticas y sentirse con pocos atributos o poco atractivos. Seguidamente, en la categoría Autoestima Social se obtuvo una media de 4,78 lo cual indica un nivel bajo. Es decir, los sujetos del estudio se perciben con poca aceptación y receptividad social, con poca capacidad para ser exitosos ante cualquier situación de este tipo, por ende, inseguros en sus relaciones interpersonales, lo que podría dificultar el establecer vínculos. Por otra parte, en la dimensión de Autoestima familia-hogar la puntuación fue baja obteniéndose una media de 4,17. Lo cual indica que los sujetos se perciben poco aceptados, considerados y respetados por parte de su familia, albergando sentimientos de minusvalía frente a ellos. Por otra parte, la autoestima Escolar-Laboral de los sujetos puntuó 5,98 situándose en nivel normal con tendencia a un nivel alto.

Este resultado indica que los sujetos se autoperciben con capacidad normal (con tendencia alta) para rendir bien, aprender, estar a gusto y ajustarse a las situaciones académico-laborales. Por último, se obtuvo un indicador normal total general de autoestima (34.27). Lo cual 
hace referencia a que en medida estándar, los sujetos "son optimistas, creativos y capaces de enfrentar la crítica, aunque con tendencia a la inseguridad al autoevaluarse" (Coopersmith, 1981 citado por
Villasmil 2010, p. 35). Por otra parte, cabe anotar, que el resultado obtenido en el factor mentira fue significativamente confiable (3.56), valor indicativo de bajo fingimiento en sus respuestas.

Tabla 2

Estadística descriptiva de la variable autoestima

\begin{tabular}{|c|c|c|c|c|c|c|}
\hline Parámetros & $\begin{array}{c}\text { cooper } \\
\text { G }\end{array}$ & $\begin{array}{c}\text { cooper } \\
\text { S }\end{array}$ & $\begin{array}{c}\text { cooper } \\
\text { FH }\end{array}$ & $\begin{array}{c}\text { cooper } \\
\mathrm{M}\end{array}$ & $\begin{array}{c}\text { cooper } \\
\text { EL }\end{array}$ & $\begin{array}{l}\text { Cooper } \\
\text { General }\end{array}$ \\
\hline Muestra & 41 & 41 & 41 & 41 & 41 & 41 \\
\hline Perdidos & 0 & 0 & 0 & 0 & 0 & 0 \\
\hline Media & 15,78 & 4,78 & 4,17 & 3,56 & 5,98 & 34,27 \\
\hline Error típ. de la media & ,677 & ,233 & ,239 & ,237 & ,642 & 1,087 \\
\hline Desv. típ. & 4,333 & 1,492 & 1,531 & 1,517 & 4,108 & 6,961 \\
\hline Mínimo & 7 & 2 & 0 & 0 & 2 & 18 \\
\hline Máximo & 23 & 8 & 7 & 6 & 25 & 50 \\
\hline
\end{tabular}

Nota. Fuente: Análisis de datos en Statistical Package for the Social Sciences

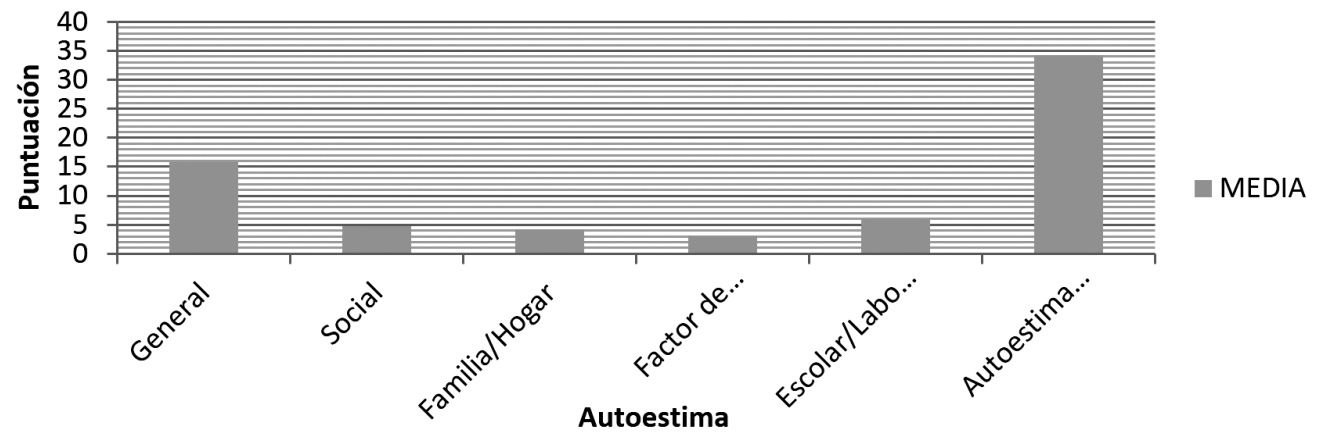

Figura 1. Puntuaciones medias del Inventario de Coopersmith

Fuente: Autores

En la Tabla 3 se observan la media, los puntajes mínimos y máximos, el error típico de la media y la desviación típica de la Escala de Estrategias de Coping Modificada aplicada a la muestra. A nivel general, los factores de la Escala, tuvieron un índice de confiabilidad igual a .66. En cuanto a las estrategias de afrontamiento, las más utilizadas por los miembros del estudio y en las cuales se encontraron puntuaciones medias con tendencia significativa a niveles altos fueron las estrategias de Solución de Problemas, Religión, Reevaluación positiva. 
Seguidas de estrategias de afrontamiento de Búsqueda de Apoyo Profesional, Evitación cognitiva y emocional, la cuales se situaron en niveles netamente medios. De otro lado, la estrategia que obtuvo la puntuación más baja de toda la escala fue: Expresión de la Dificultad, junto con Autonomía y Búsqueda de Apoyo Social. En un nivel medio de puntuación y utilización se encontraron las estrategias de Espera, Reacción Agresiva y Negación. A manera de información, se presenta en la Figura 2 los puntajes promedio de la Escala de Estrategias de Coping Modificada (EECM) de 69 ítems, validada en Colombia por Londoño, et. al., (2006) y aplicada a la muestra de personas privadas de la libertad.

Tabla 3.

Estadística descriptiva de la variable estrategia de afrontamiento

\begin{tabular}{|c|c|c|c|c|c|c|c|c|c|c|c|c|}
\hline & $\mathrm{SP}$ & BAS & ESP & REL & $\mathrm{EE}$ & BAP & $\mathrm{RA}$ & EC & $\mathrm{RP}$ & EDA & NEG & AUT \\
\hline Válidos & 41 & 41 & 41 & 41 & 41 & 41 & 41 & 41 & 41 & 41 & 41 & 41 \\
\hline Perdidos & 0 & 0 & 0 & 0 & 0 & 0 & 0 & 0 & 0 & 0 & 0 & 0 \\
\hline Media & 70,20 & 50,78 & 56,39 & 71,12 & 58,46 & 65,66 & 52,29 & 59,49 & 69,95 & 37,32 & 57,24 & 49,07 \\
\hline $\begin{array}{c}\text { Error típ. de } \\
\text { la media }\end{array}$ & 4,355 & 4,952 & 5,284 & 3,559 & 4,521 & 5,143 & 6,884 & 5,178 & 3,863 & 3,741 & 4,643 & 4,706 \\
\hline Desv. típ. & 27,885 & 31,708 & 33,837 & 22,789 & 28,951 & 32,932 & 30,660 & 33,156 & 24,737 & 23,957 & 29,731 & 30,133 \\
\hline Mínimo & 5 & 1 & 1 & 10 & 3 & 1 & 5 & 1 & 18 & 1 & 1 & 1 \\
\hline Máximo & 99 & 99 & 99 & 99 & 99 & 99 & 99 & 99 & 99 & 99 & 99 & 95 \\
\hline
\end{tabular}

Nota. Fuente: Análisis de datos en Statistical Package for the Social Sciences

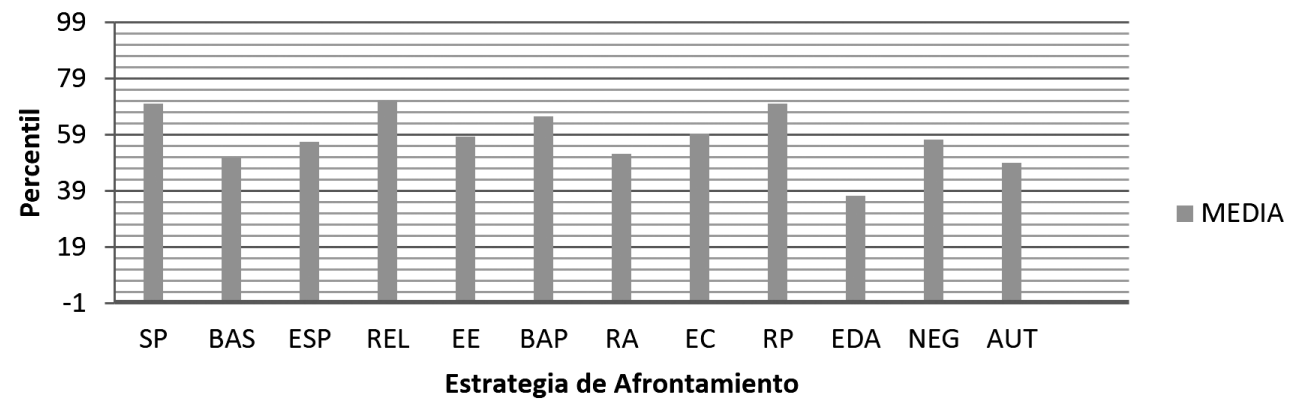

Figura 2. Puntuaciones medias de la Escala de Estrategias de Coping Modificada (ECC-M) de la muestra

Fuente: Autores

Con respecto a los resultados de la aplicación del Coeficiente de Correlación de Pearson a todas las variables propuestas, se encontró que hubo correlaciones altas positivas y negativas ( $p>.5)$ entre algunas de las estrategias de afrontamiento de la Escala EEC-M entre sí. Además se observaron 
correlaciones negativas significativas $(p<.05)$ entre la autoestima total general y las estrategias de afrontamiento
Espera, Evitación Emocional, Reacción Agresiva y Negación, como es de observar en la Tabla 4.

Tabla 4.

Correlación Entre las Diferentes Estrategias de Afrontamiento de la Escala EEC-M y el Inventario de Autoestima de Coopersmith.

\begin{tabular}{|c|c|c|c|c|c|c|c|c|c|c|c|c|c|}
\hline $\mathrm{EA}$ & SP & BAS & ESP & REL & $\mathrm{EE}$ & RAP & RA & EC & $\mathrm{RP}$ & EDA & NEG & AUT & $\begin{array}{c}\text { AUTOESTIMA } \\
\text { TOTAL } \\
\text { GENERAL }\end{array}$ \\
\hline $\mathrm{SP}$ & & $.332^{*}$ & & $.412^{\star \star}$ & & & & & $.650^{\star *}$ & & & & \\
\hline BAS & $.332^{\star}$ & & & $.347^{*}$ & & $.527^{\star \star}$ & & & $.387^{\star}$ & & & $-.346^{*}$ & \\
\hline ESP & & & & $.315^{*}$ & $.459^{\star \star}$ & & & $.578^{\star *}$ & & $.309^{\star}$ & $.510^{\star *}$ & $.323^{*}$ & $-.432^{* *}$ \\
\hline REL & $.412^{\star *}$ & $.347^{*}$ & $.315^{\star}$ & & & $.424^{\star \star}$ & & $.330^{*}$ & $.487^{\star \star}$ & & & & \\
\hline EE & & & $.459^{\star \star}$ & & & & $.453^{\star *}$ & $.596^{\star *}$ & & $.498^{\star \star}$ & $.474^{\star *}$ & $.326^{\star}$ & $-.453^{\star *}$ \\
\hline RAP & & $.527^{\star \star}$ & & $.424^{\star *}$ & & & & & $.421^{\star \star}$ & & & & \\
\hline $\mathrm{RA}$ & & & & & $.453^{\star *}$ & & & & & $.461^{\star \star}$ & & & $-.456^{\star *}$ \\
\hline EC & & & $.578^{\star *}$ & $.330^{\star}$ & $.596^{\star *}$ & & & & $.379^{*}$ & & $.664^{* *}$ & & \\
\hline $\mathrm{RP}$ & $.650^{* *}$ & $.387^{*}$ & & $.487^{\star *}$ & & $.421^{* *}$ & & $.379^{*}$ & & & & & \\
\hline EDA & & & $.309^{\star}$ & & $.498^{* *}$ & & $.461^{* *}$ & & & & & $.410^{\star *}$ & \\
\hline NEG & & & $.510^{\star \star}$ & & $.474^{\star \star}$ & & & $.664^{\star \star}$ & & & & & $-.319^{\star}$ \\
\hline AUT & & $-.346^{*}$ & $.323^{*}$ & & $.326^{\star}$ & & & & & $.410^{\star *}$ & & & \\
\hline
\end{tabular}

Nota. Fuente: Análisis de datos en Statistical Package for the Social Sciences

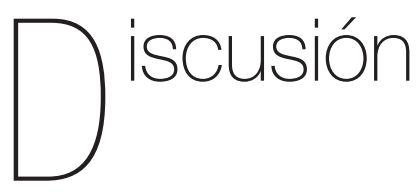

La autoestima, reviste de gran importancia para la salud psicológica de los sujetos al estar directamente relacionada con la planeación del futuro, las estrategias para enfrentar situaciones estresantes, la toma de decisiones, la solución de problemas, las relaciones interpersonales, la autoevaluación de sus capacidades y habilidades (Eisenberg \& Patterson, 1981 citado por Naranjo, 2007). Bajo este postulado, los niveles bajos en la misma, pueden ocasionar una interferencia negativa, haciendo que durante el ciclo vital no puedan desarrollarse plenamente. Proposición, coincidente con Maslow (1968) en lo referente a la autoestima y la autorealización.

Ahora bien, aunque Echeburrúa y Corral (1988) y Pérez y Redondo (1991) han descrito que las personas privadas de la libertad exhiben niveles bajos de autoestima; en este estudio, se estableció que los sujetos muestran características de autoestima en el rango de lo normal. Entre éstas estan valoración, confianza, seguridad, respeto de sí mismo, optimismo, creatividad y capacidad para enfrentar la crítica. Sin embargo, presentan tendencia a experimentar desánimo, tristeza, 
preocupación, sensibilidad a las críticas y a sentirse con pocos atributos o poco atractivos, lo que posiblemente se encuentre relacionado con la condición de "convictos".

Por otra parte, se observó que con el ánimo de mantener la valoración de sí mismos, los sujetos privados de la libertad, procuran enfrentarse a la situación de prisionización, resistir y salir adelante, a través de la vinculación a programas válidos para redención de pena ofrecidos por el centro de reclusión, bien sean de trabajo, estudio o enseñanza; elementos contemplados como medios terapéuticos que contribuyen a restablecer o mantener una identidad positiva, sentido de pertenencia y/o sentido de ser útiles. Lo cual guarda coherencia con los datos obtenidos en la escala correspondiente a autoestima laboral, en la que se refleja a unos sujetos con capacidad normal, tendencia alta para rendir bien, aprender, estar a gusto y ajustarse a las situaciones académico-laborales. Resultados validados por Viano, Villagra y Martinez (2008) quienes afirman que:

\section{Entre los principales beneficios para quienes se insertan en este proceso (principalmente laboral, aunque también aplica para lo escolar) dirigido a adquirir habilidades y competencias para mejorar su empleabilidad futura, está el aumento de la satisfacción personal, la disminución de la inseguridad y el fortalecimiento de la autoestima"(p. 4).}

No obstante, debido a la propia situación de condenados y a los efectos que produce el encierro, e incluso debido a la situación ambiental, no dejan de experimentar tendencia al afecto negativo, aunque su autoestima general y autoestima escolar-laboral se mantenga dentro de límites normales. Por lo anterior, el mantener la valía personal, aunada a la vinculación ocupacional de los sujetos favorece positivamente su proceso de prisionización, abriendo posibilidades al apoyo social, crecimiento personal, cognoscitivo, laboral e incluso económico, a pesar de encontrarse en medio ambiente disruptivo.

No así, la autoestima social describe sujetos que se perciben con poca aceptación y receptividad, con poca capacidad para triunfar ante cualquier situación, por ende, hay inseguridad en sus relaciones interpersonales, estableciendo limitados lazos sociales. Situación que se desprende debido al peso que le asiste a la estigmatización sobre la persona privada de la libertad, la desaprobación y marginación social; al culparlo y excluirlo por haber afectado o puesto en riesgo los intereses vitales de la sociedad. Sumado a la influencia de sentimientos de vergüenza, culpa, desesperanza-ansiedad frente al futuro, pérdida de amigos, familiares y rutinas socio-labores que llevaban antes de su ingreso al centro de reclusión.

De igual manera, se perciben poco aceptados, considerados y respetados por parte de su familia, albergando sentimientos de minusvalía frente a ellos, principalmente por: no poder aportar a sus necesidades, rutinas, tareas, sentir que están abandonados, la pérdida de independencia que los lleva a solicitar ayuda para su sostenimiento, al estigma social y en ocasiones al peso que acarrea haber cometido algún delito específicamente en contra de miembros de la familia. 
Con respecto a las estrategias de afrontamiento, los resultados obtenidos en estudios hechos por Kitchner y Mohino (2003) y Mikulic y Crespi (2004) indican un predominio de respuestas de afrontamiento de tipo evitativas en las personas privadas de la libertad, contrario a lo evidenciado en el presente estudio, donde los sujetos evaluados tienden al uso de estrategias de Solución de problemas, Religión y Reevaluación positiva. Aunque, se presentó un uso menor de la estrategia de Expresión de la Dificultad de Afrontamiento y la autonomía, esta última encuentra coincidencia con el planteamiento de Perez y Redondo (1991) cuando mencionan que los sujetos privados de la libertad son menos autónomos; lo que trae como consecuencia el desarrollo principalmente de locus de control externo.

De igual manera la búsqueda de apoyo social aparece como de bajo uso, guardando relación con los resultados evidenciados anteriormente en la escala de autoestima social.

Los sujetos del estudio utilizan en un nivel estándar las estrategias de Espera, Reacción Agresiva y Negación. Finalmente, se pudo establecer, a partir de las variables Autoestima y Estrategias de Afrontamiento (Negación, Reacción Agresiva, Evitación Emocional y Espera) que existe una correlación principalmente negativa entre estas dos variables. Por ende, a medida que la autoestima aumenta en los sujetos, estas estrategias disminuyen. Es así como al hacer la correlación respectiva se evidenció en primer lugar, que a medida que la Estrategia de Afrontamiento de Negación disminuye aumenta la variable autoestima y viceversa. Esto, coincidente con Branden (1995) quien afirma que, el aprecio de sí mismo permite a la persona enfrentarse a las vicisitudes cotidianas con mayor seguridad. Por tanto, se afirma para los sujetos del estudio que al hacer frente a la mayoría de situaciones estresantes que se le presentan habrá menor negación del asunto, lo que deriva en el incremento o conservación de la autoestima y autoeficacia. En segundo lugar, se observó que mientras la Estrategia de Afrontamiento de Reacción Agresiva asciende, la variable autoestima mengua y viceversa. Lo que de manera general concuerda con lo propuesto por Walker y Bright (2009 citados por Loinaz, Echeburrúa \& Ullate, 2013) quienes manifiestan que hay una fuerte asociación entre baja autoestima y el significativo aumento y gravedad de actos de violencia. En tercer lugar, se evidenció que a menor Evitación Emocional y Espera la variable Autoestima aumenta y viceversa. Por ello, se infiere que al hacer menor uso estas estrategias que entrañan algún tinte de pasividad, habrá tendencia en los sujetos a considerar en menor medida que no se puede hacer mucho en cuanto a la situación, que la situación se resolverá por si sola o a optar por inhibir las emociones que suscita el evento estresante. No obstante, tendrá que salir a relucir el empoderamiento, la autoeficacia y confianza en sí mismo con lo cual enfrentará de manera eficaz cualquier situación que le genere estrés.

A manera de conclusión tenemos que se pudo establecer que si bien es cierto que el contexto de reclusión es adverso en sí mismo, también lo es que los programas de tratamiento penitenciario ofrecidos, coadyuvan a mantener o restablecer, conservar la autoestima en los sujetos. Sin omitir por supuesto que la autoestima General, Social, y Familiar se ve afectada sin duda alguna por el delito cometido y 
el ingreso a un centro carcelario. Sin embargo, podría decirse que los programas de tratamiento penitenciario laborales $\mathrm{o}$ educativos, se constituyen en un medio terapéutico capaz de afectar de manera positiva la autoestima del personal de internos que alberga la prisión. Por otra parte se pudo observar que las estrategias de afrontamiento poco pasivas (SP, REL, $\mathrm{RP}, \mathrm{BAP})$ son utilizadas por este grupo de personas frente a las situaciones estresantes que se les presentan. Por último, la relación entre autoestima y las estrategias de Afrontamiento (NEG, RA, EE, ESP) es significativa e inversamente proporcional.

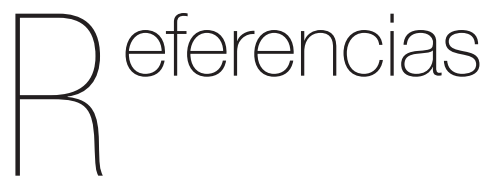

Branden, N. (1995). Los seis pilares de la autoestima. Madrid: Ediciones Paidós Ibérica, S. A.

Cava, M., Musitu, G., \& Vera, A. (2000). Efectos directos e indirectos de la autoestima en el ánimo depresivo. Revista Mexicana de Psicología, 17(2), 151-161.

Chorot, P., \& Sandín, B. (1993). Escala de estrategias de coping revisada (EEC-R). Editorial UNED. Madrid: España.

Coopersmith, S. (1981). The antecedents of self-steem.Consulting Psycologist Press. Palo Alto: California.

Echeburrúa, E., \& Corral, G. (1988). E tratamiento psicológico en las instituciones penitenciarias, alcance y limitaciones. Cuaderno del Instituto Vasco de Criminología San Sebastian, 1, 179-190.
Fernández, B, (2006). La cárcel en el entorno familiar, estudio de las repercusiones del encarcelamiento sobre las familias: problemáticas y necesidades. Observatori del Sistema Penal i els Drets Humans, Universitat de Barcelona. Barcelona: España.

Fernández, E., \& Palmero, F. (1999). Emociones y salud. Barcelona: Ariel.

Herrera, M., \& Expósito, F. (2010). Una vida entre rejas: Aspectos psicosociales de la encarcelación y diferencias de género. Revista Intervención Psicosocial, 19 (3), 235-241.

Kirchner, T., \& Mohino, S. (2003). Estrategias de coping y conducta autolesiva en jóvenes reclusos. Revista Psiquiatría Facultad de Medicina Barna, 30(2), 77-85.

Lázarus, R., \& Folkman, S. (1986). Estrés y Procesos cognitivos. Barcelona: Editorial Martínez Roca.

Loinaz, I., Echeburúa, E., \& Ullate, M. (2012). Estilo de apego, empatía y autoestima en agresores de pareja. Revista Terapia Psicológica, 30(2) 61- 70.

Londoño, N., Henao, G., Puerta, I., Posada, S., Arango, D., \& Aguirre, D. (2006). Propiedades psicométricas y validación de la escala de Estrategias de Coping Modificada (EEC-M) en una muestra colombiana. Universitas Psychologica, 5, 327-349.

Marcuello, C., \& García, J. (2011). La cárcel como espacio de de-socialización ciudadana: ¿fracaso del sistema penitenciario español? Portularia, XI, 1, 49-60. 
Martín, M., Jiménez, M., \& Fernández- Abarcal, E. (1997). Estudio sobre la Escala de Estilos y Estrategias de Afrontamiento. Revista Electrónica de Motivación y Emoción, 3(4), 58-69.

Maslow, A. (1982). La amplitud potencial de la naturaleza humana. México, D.F: Editorial Trillas.

Mikulic, I, \& Crespi, M. (2004). Contexto carcelario: un estudio de los estresores y las respuestas de afrontamiento en detenidos primarios y reincidentes. XII Anuario de Investigaciones, Universidad de Buenos Aires. Buenos Aires: Argentina.

Montenegro, V., \& Ortega, P. (2008). Intervención psicoterapéutica cognitivoconductual, para optimizar el nivel de adaptación carcelaria de las internas imputadas y sentenciadas del Centro de Rehabilitación Social de Machala. Tesis de pregrado publicada. Universidad tecnológica San Antonio Machala, San Antonio Machala: Ecuador.

Monteverde, S. (1982). Las cárceles y prisiones en la segunda mitad del siglo XVIII y comienzos del XIX. Aportes teóricos y penales de Jeremías Bentham. Revista de Estudios Histórico-Jurídicos, 20. doi: 10.4067/ S0716-54551998000200007

Naranjo, M. (2007). Autoestima: un factor relevante en la vida de la persona y tema esencial del proceso educativo. Revista Actualidades Investigativas en Educación, 7(3) 1-27.

Pérez, E., \& Redondo, S. (1991). Efectos psicológicos de la estancia en prisión. Revista Papeles del Psicólogo on line, 41.
Quintanilla, R., Valadez, I., Valencia, S., \& González, J. (2005). Estrategias de afrontamiento en pacientes con tentativa suicida. Revista Investigación en Salud, 7(2), 112-116.

Ruiz, J. (1999). Estrés en prisión y factores psicosociales. Revista Colombiana de Psicología, 8, 120-130.

Ruiz, J. (2007). Síntomas psicológicos, clima emocional, cultura y factores psicosociales en el medio penitenciario. Revista Latinoamericana de Psicología, 39(3) 547-561.

Santrock, J. (2002). Psicología de la educación. México, D.F: Mc Graw Hill.

Segovia, J. (2005). Consecuencias de la prisionización. Cuaderno de Derecho Penitenciario. Madrid: Universidad Pontifica de Salamanca.

Smith, J., \& Hogan, G. (1973). Criminal Law. London: Burke worth.

Viano, C., Villagra, C., Martínez, F. (2008). Revista electrónica Debates Penitenciarios, 8. Centros de Estudios en Seguridad Ciudadana. Universidad de Chile. Santiago: Chile.

Villasmil, J. (2010). El autoconcepto académico en estudiantes universitarios resilientes de alto rendimiento: un estudio de casos. Tesis de doctorado. Mérida: Venezuela.

Wheeler, S. (2007). Socialization in correctional communities. Brithish Psychological Review, 26,15, 267-282.

Zamble, E., \& Porporino, F. (1988). Coping, Behavior, and adaption in prison inmates. New York: Springe 